\title{
Shape Control of Doped Semiconductor Nanocrystals (d-Dots)
}

\author{
Ranjani Viswanatha', David M. Battaglia' ${ }^{2}$, Mark E. Curtis ${ }^{3,4}$, Tetsuya D. Mishima ${ }^{3,4}$, Matthew B. Johnson ${ }^{3,4}$, \\ and Xiaogang Peng ${ }^{1,3}(\bowtie)$ \\ ${ }^{1}$ Department of Chemistry \& Biochemistry, University of Arkansas, Fayetteville, AR 72701, USA \\ ${ }^{2}$ NN-Labs LLC, Fayetteville, AR 72703, USA \\ ${ }^{3}$ Joint MRSEC at the University of Oklahoma and the University of Arkansas, Fayetteville, AR 72701, USA \\ ${ }^{4}$ Homer L. Dodge Department of Physics and Astronomy, University of Oklahoma, Norman, OK 73019, USA \\ Received: 12 May 2008/ Revised: 5 June 2008/Accepted: 5 June 2008 \\ (C)Tsinghua Press and Springer-Verlag 2008
}

\begin{abstract}
Formation of $\mathrm{Mn}^{2+}$-doped $\mathrm{ZnSe}$ quantum dots (Mn:ZnSe d-dots) with both branched and nearly spherical shapes has been studied. Structure analysis indicates that the $\mathrm{Mn}^{2+}$ dopants were localized in the core of a branched nanocrystal. The growth of branched d-dots, rather than spherical ones, was achieved by simply varying the concentration of two organic additives, fatty acids, and fatty amines. The photoluminescence properties of the branched nanocrystals were explored and compared with those of the nearly spherical particles.
\end{abstract}

\section{KEYWORDS}

Doped quantum dot (d-dot), shape control, photoluminescence

Doping plays the central role in many areas of modern semiconductor science and technology, such as light-emitting diodes, solar cells, and spintronics. In the field of nanomaterials, it is expected that doped nanostructures will also have unprecedented properties. For instance, semiconductor nanocrystals doped with transition metal ions (d-dots) may offer a new generation of emissive nanocrystals $[1,2]$, with high efficiency, high temperature stability [3], zero self-quenching due to absence of self-absorption and Foster-energy transfer, and furthermore they are heavy metal-free. Efforts on developing semiconductor nanostructures doped with magnetic centers are also being actively pursued with the aim of developing nanomaterials with both semiconductor and magnetic properties [4-8]. Recent progress in synthetic chemistry allows essentially all the colloidal nanocrystals in a sample to be doped with a small amount of a dopant [2,9-12]. Success of the doping chemistry, however, has been limited to nearly spherical d-dots. This report will demonstrate that, by simply varying the concentrations of organic additives - fatty acids and fatty amines - formation of branched Mn-doped ZnSe (Mn:ZnSe) d-dots with the center of the branched nanocrystals being doped is possible.

Shape control of high quality colloidal semiconductor nanocrystals has been studied extensively in recent years [13-15]. The anisotropic growth of thermodynamically meta-stable shapes, such as the

Address correspondence to xpeng@uark.edu 
formation of elongated shapes in II-VI semiconductor nanocrystals with hexagonal and zinc-blende crystal structures, is governed mainly by two factors. The first requirement is that a few of the facets of a given nanocrystal become chemically more active than the others, which is usually achieved by discriminating interactions between organic ligands and the surface atoms. The second requirement is that the monomer activity of the system must be sufficiently high to offer the metastable nanocrystals a compatible solution environment [13]. The results described below reveal that these two factors can be controlled independently in the formation of metastable shapes of d-dots by simply varying the concentrations of fatty amines and fatty acids usually needed for the formation of high quality ZnSe host [16] and related doped nanocrystals [2].

The synthetic scheme for the shape-controlled $\mathrm{Mn}: \mathrm{ZnSe}$ d-dots followed a modified procedure reported previously [10] (see Experimental section for details). Briefly, manganese fatty acid salts, zinc fatty acid salts and elemental selenium were used as the manganese, zinc and selenium precursors, respectively. The solvent used was 1-octadecene (ODE), a common non-coordinating solvent. The concentrations of two organic additives, fatty amines and fatty acids, were varied in order to manipulate the surface passivation and the activity coefficients of the monomers.

Controlling the reactivity of the zinc precursor was shown in an earlier work to be the key to ensuring a uniform coating of the ZnSe layers on the MnSe core [10]. The reactivity of the $\mathrm{Zn}$ precursor was kept sufficiently high to ensure overcoating of the precursor MnSe dots but low enough to avoid homogeneous nucleation of ZnSe nanocrystals. This was achieved by maintaining a balance between the fatty amines, as activation reagents, and the fatty acids, as inhibitors. It should be pointed out that fatty amines are thought to be activation reagents in this system and in other related systems.

Figure 1 shows representative TEM images of the nanocrystals formed in the current system. The small quasi-spherical d-dots (Fig. 1(a)) were synthesized following the standard core-doping strategy [2]. Addition of aliquots of a zinc fatty acid salt precursor dissolved in ODE with given amounts of amine and acid to the MnSe particles enabled the growth of the host semiconductor (pure ZnSe) overcoating layer. The resulting d-dots obtained with different amounts of fatty acid but a fixed amount of amine are depicted in the right-hand column in Fig. 1. The left-hand column in Fig. 1 shows the TEM pictures of the resultant d-dots formed with different amounts of amine and a fixed concentration of fatty acid.

The presence of free fatty acids is known to slow down the incorporation of the fatty acid salts [17], which act as the Zn precursor in the current system, into the nanocrystals. When the fatty acid concentration was low, the Zn precursor reacted

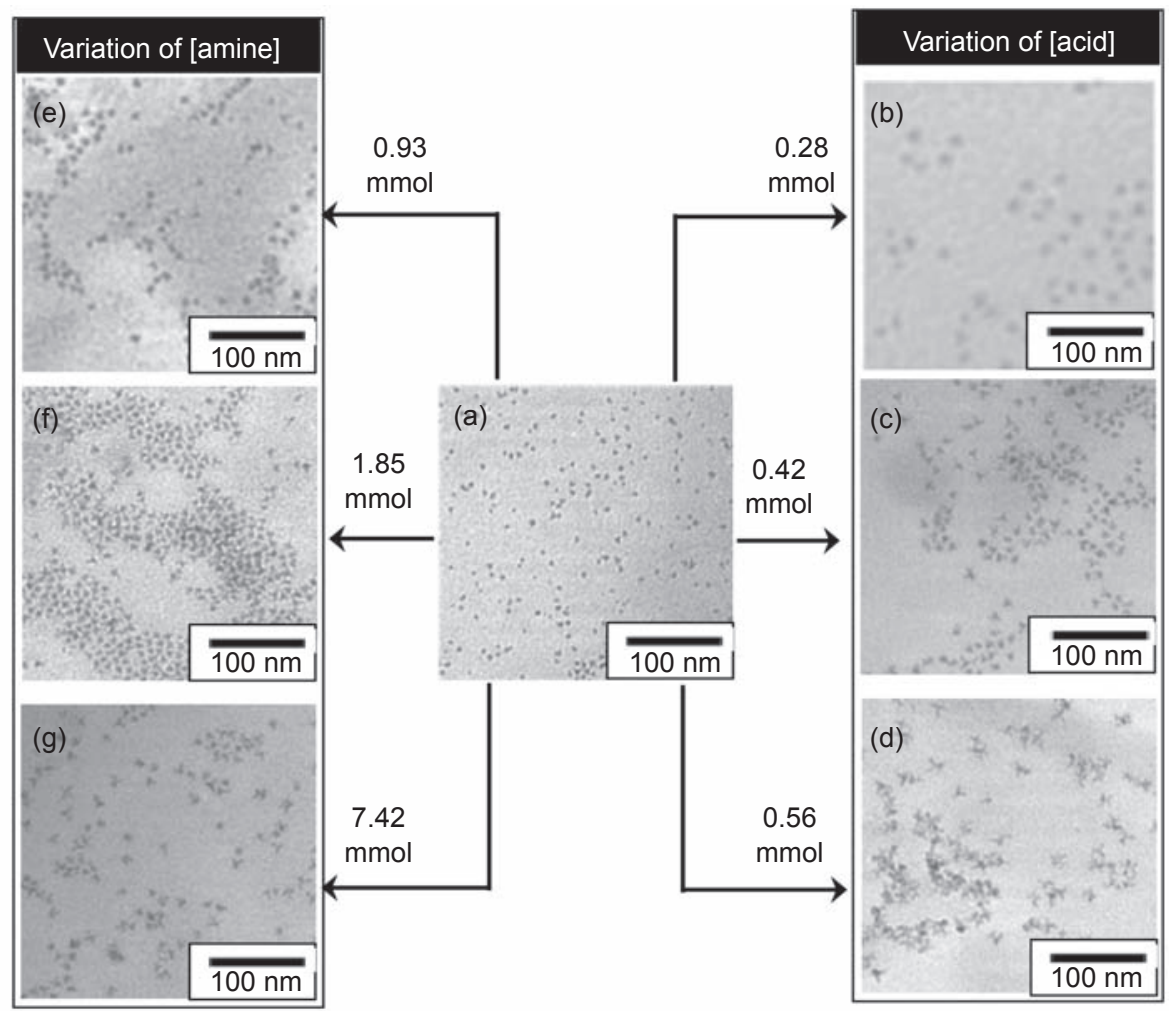

Figure 1 TEM images of Mn:ZnSe d-dots formed by varying the amounts of stearic acid (right) and octadecylamine (left) 
completely within the time period for each reaction cycle. As a result, large spherical d-dots were obtained (Fig. 1(b)). With increasing concentration of the fatty acid, the deposition of the zinc precursor onto the surface of the existing d-dots slowed down, and the precursors were thus accumulated in the system after each injection of the precursor mixture. As a result, the high monomer concentration at the end of each injection cycle offered the system a high chemical potential environment for the growth of branched d-dots. Consistent with this hypothesis, the degree of branching of the resulting d-dots increased as the concentration of the fatty acid increased (Fig. 1, right-hand column). As pointed out above, similar observations were reported earlier for intrinsic II-VI semiconductor nanocrystal systems.

Fatty amines were originally exploited as the activation reagents for the formation of intrinsic ZnSe nanocrystals using zinc fatty acid salts as the zinc precursor [16]. It was observed that zinc fatty acid salts are not very reactive at the typical reaction temperatures employed for the formation of $\mathrm{ZnO}$ [18] and ZnSe nanocrystals [16]. Fatty amines activate the zinc fatty acid salts by the formation of amides. However, amines are also known to act as surface ligands for the resulting II-VI semiconductor nanocrystals. Thus, an excess of amines may not only activate the zinc precursors, but also affect the ligand distribution on the surface of the nanocrystals. The latter effect, in principle, may in turn change the morphology of the resulting d-dots by breaking the symmetry. However, up to this point, there has been no report of amine-modulated shape control for IIVI semiconductor nanocrystals in non-coordinating solvents. Conversely, formation of elongated IIVI semiconductor nanocrystals in coordinating solvents, such as nearly pure amines, was reported even in the early stages of the study of shapecontrolled synthesis of intrinsic semiconductor nanocrystals [16]. This contrast is probably a result of the highly dynamic nature of the amine ligands, as reported recently, which indicates that the surface coordinating effects of amine ligands should be more observable for a high concentration of amines [19]. This argument is consistent with our results in Fig. 1 (right-hand column). In comparison to the relatively small increases in concentration of fatty acid required to produce branched dots (left-hand column, Fig. 1), similar branched d-dots could only be formed with a substantially higher concentration of amine - roughly 5-10 fold higher than that of the fatty acid.

To further confirm the above hypothesis, we compared the growth pattern of d-dots using fatty amines with different chain lengths (octadecylamine (ODA) vs decylamine (DA)). It has been previously observed that the surface ligand dynamics in the amine-CdSe ligand-nanocrystal system changed drastically around the boiling point of the fatty amine employed [19]. This is so because the bonding dynamics of fatty amines, as weak ligands for II-VI semiconductor nanocrystals, are dominated by the ligand-ligand interactions on the surface. Consequently, as the temperature is raised above the boiling point (bp), the ligand dynamics are drastically accelerated from a quasi-liquid state to a quasi-vapor state. Since the chain length of the amine determines their boiling points, at any given temperature the surface amine ligand becomes more dynamic as the chain length decreases. Hence, if the proposed mechanism is valid in the present case, we should observe a ligand chain length-dependent morphology change of the resulting d-dots.

To compare the amine chain length effect, Mn: $\mathrm{ZnSe}$ d-dots were grown under the same conditions for the two different amines. After addition of the $\mathrm{Zn}$ precursor, equimolar amounts of either decylamine (DA) (bp $217^{\circ} \mathrm{C}$ ) or octadecylamine (ODA) (bp $361{ }^{\circ} \mathrm{C}$ ) were added into the reaction flask. The overcoating temperature was similar that usually employed [10], between $275{ }^{\circ} \mathrm{C}$ and $290{ }^{\circ} \mathrm{C}$, which is higher than the boiling point of DA and lower than the boiling point of ODA. The final d-dots produced in the two comparative reactions were examined using TEM (Fig. 2). The morphologies of the resulting d-dots in the two cases were different. The reaction with DA (low boiling point amine) yielded nearly spherical nanocrystals, whilst under identical conditions, the reaction with ODA (high boiling point amine) produced branched ones. This is consistent with the proposed surface ligand dynamic argument.

The branched d-dots were found to be single crystal in nature by high-resolution transmission 
electron microscopy (HRTEM). An HRTEM image of a typical branched d-dot is shown in Fig. 3(a). The crystal structure analysis of the HRTEM image suggests that the core as well as all the branches crystallized in the zinc blende structure (see a detailed lattice analysis in the Electronic Supplementary Material (ESM)). For the image in Fig. 3(a), while the core is oriented [110] in the viewing direction, two of the branches with twinning relations to the core are oriented $[-1-10]$ to the viewing direction. As for the other two branches, one has its (111) plane lying essentially along the (001) plane of the core (misoriented by $5^{\circ}$ ), while the other has its (001) plane lying essentially along the (1-
11) plane of the core (misoriented by $9^{\circ}$ ). It should be pointed out that the d-dots were not found to be regularly branched as has been previously observed for II-VI semiconductor tetrapod nanocrystals with the wurtzite crystal structure, such as CdSe and CdTe tetrapods [15].

Figure 3(b) shows a high-angle annular dark-field (HAADF) scanning transmission electron microscopy (STEM) image and the spatially resolved electronic data systems (EDS) analysis of a branched d-dot. Examples of the EDS spectra of the core (A) and branch (B) areas are provided in the ESM, and the $\mathrm{Mn}$ to $\mathrm{Zn}$ molar ratio (Mn:Zn) calculated from the background-subtracted counts are shown as an inset
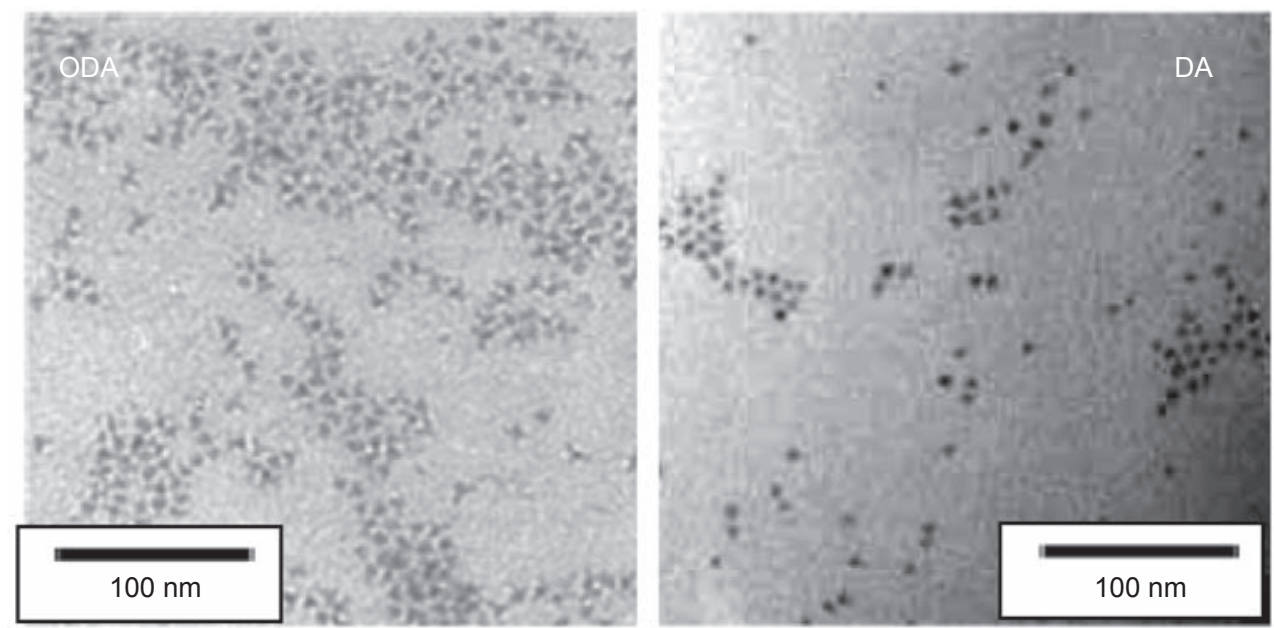

Figure 2 TEM images of the Mn:ZnSe d-dots synthesized using ODA and DA showing branched and spherical nanocrystals, respectively

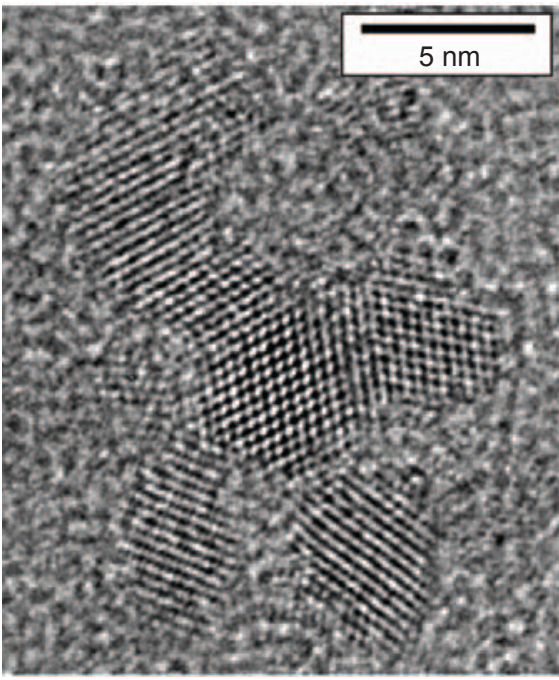

(a)

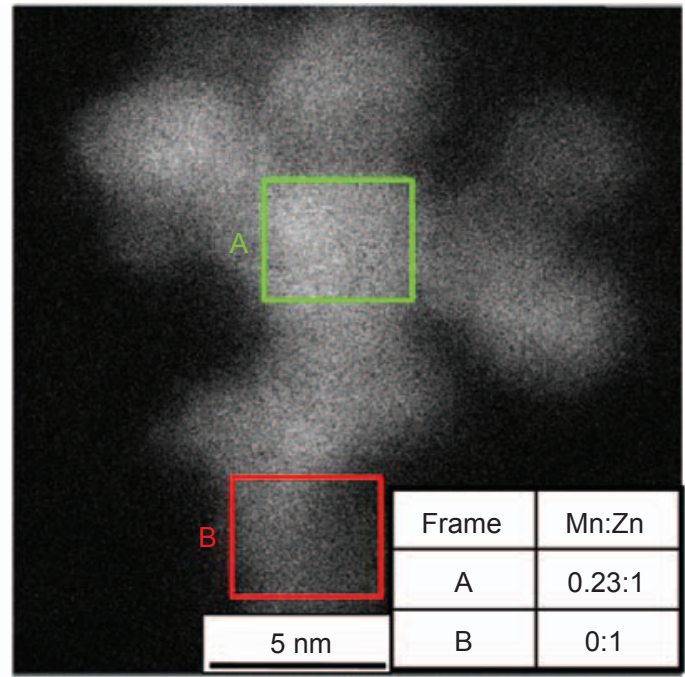

(b)

Figure 3 (a) HRTEM image of a typical branched d-dot. (b) High-angle annular dark-field (HAADF) scanning TEM image of a branched d-dot with EDS results (inset) 
in Fig. 3(b). It is noteworthy that although Mn was detected in the core area of the branched d-dot, it was not found in the branches.

The local structure of $\mathrm{Mn}$ ions in the spherical dots as well as branched particles was further probed using electron paramagnetic resonance (EPR) spectroscopy. Figure 4(a) shows the EPR spectra of the $\mathrm{Mn}$ ions in d-dots with different morphologies. The spectra exhibit well-resolved hyperfine splitting, characteristic of isolated $\mathrm{Mn}$ ions. This suggests that a significant portion of the Mn ions, at least, were not involved in strong $\mathrm{Mn}-\mathrm{Mn}$ interactions and were intercalated with the $\mathrm{Zn}$ ions. Interestingly, the

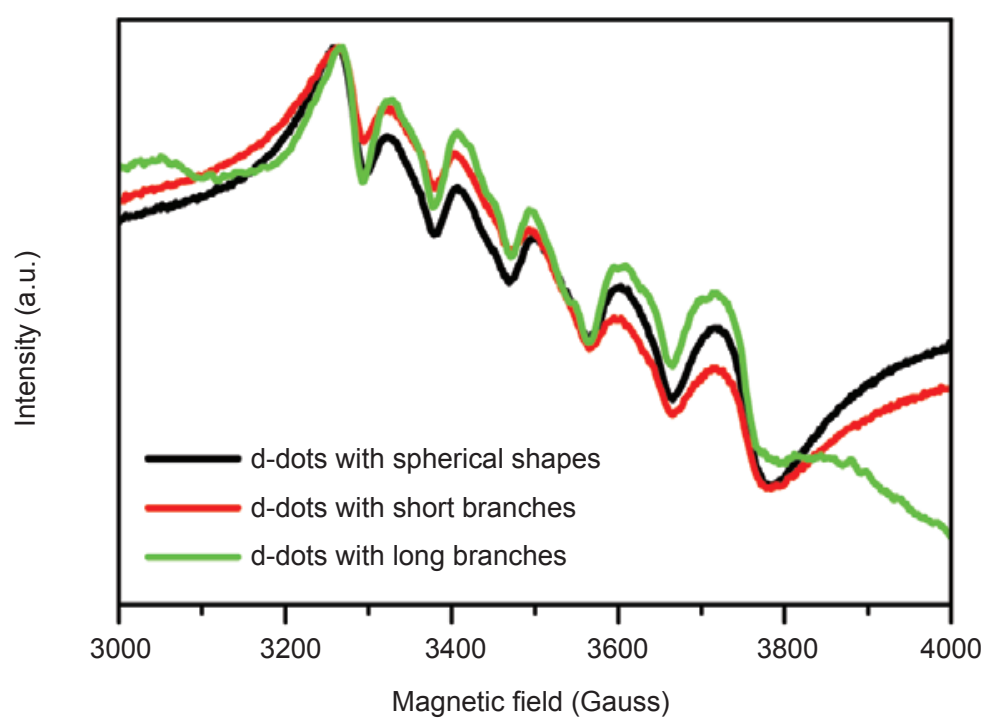

(a)

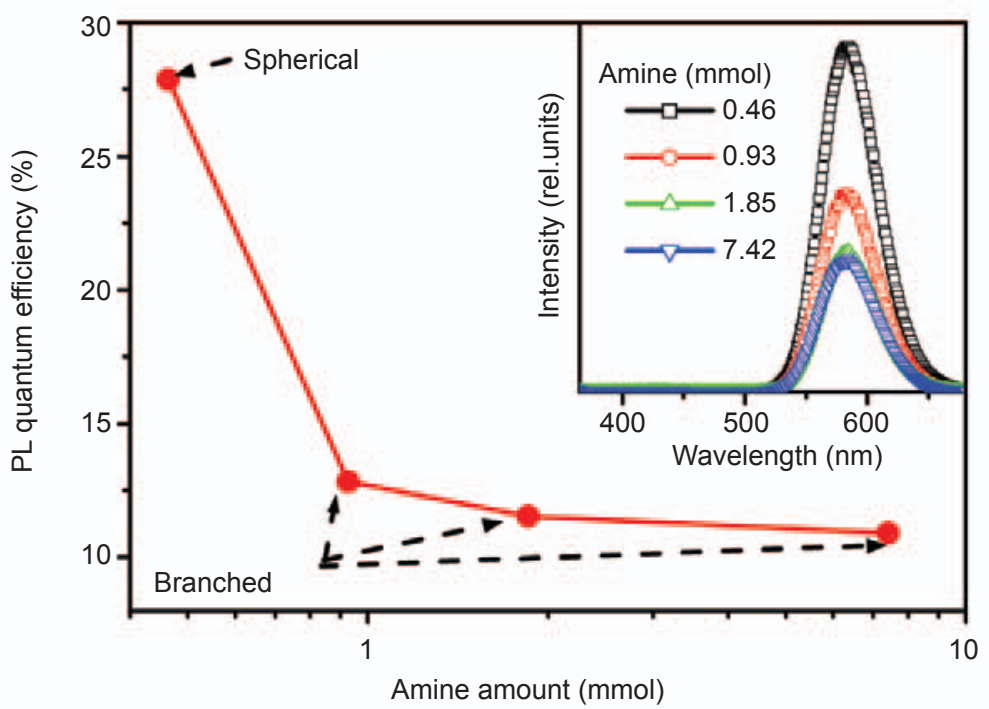

(b)

Figure 4 (a) EPR spectra of the Mn ion of different shaped d-dots. (b) PL properties of d-dots with different shapes observed hyperfine coupling constant was not the same as that of either interior-doped Mn ions with a low dopant concentration or surface-doped Mn ions in bulk samples [4]. However, EXAFS measurements (data not shown) indicated that the Mn ions in the $\mathrm{d}$-dots were interior-doped by replacement of some of the zinc sites in the ZnSe lattice. The reason for this interesting EPR spectrum is not known and deserves further study. For the current work, it is important to note the close similarity of the EPR spectra for the d-dots with different shapes. This indicates that the local environment of $\mathrm{Mn}$ ions did not change during the growth process. This further implies that the Mn centers were introduced into a unique local environment in the initial stage, i.e., in the core of the d-dots. It should be noted that this result is in agreement with the spatially resolved EDS discussed earlier (Fig. 3(b) and the related text).

The photoluminescence (PL) spectra of d-dots with different shapes were all dominated by the Mn dopant emission and the emission from the host ZnSe nanocrystals was essentially not detectable (Fig. 4(b) (inset)). This result was expected as the branched d-dots are single crystal in nature as shown in Fig. 3(a). However, the PL quantum efficiency was found to decrease drastically as the shape of the d-dots deviated from nearly spherical (Fig. 4(b)). This observation is consistent with the structural features of the branched d-dots. When an excition is generated by photo-excitation of the host semiconductor, it must travel a reasonable distance before it can be trapped by the $\mathrm{Mn}$ centers located only in the core (Fig. 3(b)). As a result, an exciton generated in the branched d-dots has a greater chance of being trapped by the surface defects than an exciton in the nearly spherical d-dots. In addition, the surface to volume ratio for the branched d-dots is much higher than that of a nearly spherical one. These two factors are both consistent with the decreased PL quantum efficiency of the branched d-dots. 


\section{Experimental}

Materials: Zinc stearate $\left(\mathrm{ZnSt}_{2}\right)$, stearic acid (SA, $95 \%$ ), selenium powder (200 mesh, 99.999\%), manganese stearate $\left(\mathrm{MnSt}_{2}\right)$, decylamine (DA), octadecylamine (ODA, 95\%), and tributyl phosphine (TBP, 95\%) were purchased from Alpha Aesar. 1-Octadecene (ODE), was purchased from Aldrich. All chemicals were used without further purification

Stock solutions: TBPSe solution was prepared inside a glovebox by adding Se (1.9 g) to TBP (8 g). The zinc precursor solutions were prepared by dissolving $\mathrm{ZnSt}_{2}(0.36 \mathrm{~g})$ and a given amount of stearic acid in $2.4 \mathrm{~g}$ of ODE. The amount of the free acid $(y \mathrm{~g})$ for each reaction was varied depending on the shape of the particles desired as discussed in the text.

Typical synthesis of d-dots: The synthesis of the d-dots was similar to the earlier reported procedure [10]. $\mathrm{MnSt}_{2}(0.02 \mathrm{~g})$ in ODE was briefly degassed at $120{ }^{\circ} \mathrm{C}$ and heated to $280{ }^{\circ} \mathrm{C}$ under Ar flow. ODA $(0.2 \mathrm{~g})$ and TBPSe stock solution $(0.2 \mathrm{~mL})$ were heated to $70{ }^{\circ} \mathrm{C}$ and injected into the above reaction flask at $280{ }^{\circ} \mathrm{C}$. After the injection, the mixture was cooled to $260{ }^{\circ} \mathrm{C}$ and annealed for $20 \mathrm{~min}$. The reaction temperature was then set at $290{ }^{\circ} \mathrm{C}$ for ZnSe overcoating. The zinc precursor solution was warmed and $1 \mathrm{~mL}$ of the stock solution was injected into the reaction flask followed by a given amount of heated ODA or DA in ODE $(0.5 \mathrm{~g})$. The amount of the amine was determined by the targeted shape of the d-dots as discussed in the text. The temperature was then reduced to $275{ }^{\circ} \mathrm{C}$. The $\mathrm{ZnSt}_{2}$ stock solution $(1 \mathrm{~mL})$ was again injected into the reaction mixture after 10 min interval, followed by ODA or DA injection and the temperature was further decreased to $270{ }^{\circ} \mathrm{C}$. The remaining $\mathrm{ZnSt}_{2}$ solution was then injected after a 10-min annealing step. The growth process was monitored by taking aliquots for UV-visible absorption spectroscopy, PL measurements, and TEM studies. Finally, the reaction was cooled to room temperature, and the nanocrystals were precipitated using acetone.

Characterization: UV-visible spectra were recorded on an HP 8453 UV-visible spectrophotometer. Photoluminescence spectra were taken using a Spex Fluorolog-3 fluorometer. The PL quantum yield
(QY) was measured using a similar method to that described in the literature [10]. Low resolution TEM images were taken on a JEOL X-100 electron microscope using a $100-\mathrm{kV}$ accelerating voltage. Specimens were prepared by dipping a Formvarcoated copper grid into a toluene solution of the nanocrystals, and the grid with the nanocrystals was allowed to dry in air. High-resolution TEM images of branched d-dots were obtained with a JEOL 2010F TEM at $200 \mathrm{kV}$ accelerating voltage. The specimen was prepared by dispersing the samples in chloroform and pipetting them onto a copper grid with an ultrathin carbon film supported by a lacey carbon film, purchased from Ted Pella, Inc. EDS was performed using a 300-kV FEI Titan TEM at Texas Instruments by Dr. Guoda Lian, and the corresponding images were taken using high-angle annular dark-field (HAADF) STEM. The EPR spectra of the powder samples were recorded on Bruker EMX spectrometer at $9.85 \mathrm{GHz}$ frequency at room temperature.

\section{Acknowledgements}

This work was supported by the National Science Foundation and the National Institute of Health. We thank Dr. Guoda Lian and Texas Instruments for EDS measurements.

Electronic Supplementary Material: Detailed analysis of an HRTEM micrograph and EDS spectra of branched d-dots are available in the online version of this article at http://dx.doi.org/10.1007/ s12274-008-8016-5 and is accessible free of charge.

\section{References}

[1] Bhargava, R. N.; Gallagher, D.; Hong, X.; Nurmikko, A. Optical-properties of manganese-doped nanocrystals of ZnS. Phys. Rev. Lett. 1994, 72, 416-419.

[2] Pradhan, N.; Goorskey, D.; Thessing, J.; Peng, X. An alternative of CdSe nanocrystal emitters: Pure and tunable impurity emissions in ZnSe nanocrystals. J. Am. Chem. Soc. 2005, 127, 17586-17587.

[3] Kim, J. H.; Holloway, P. H. Near-infraredelectroluminescent light-emitting planar optical sources 
based on gallium nitride doped with rare earths. Adv. Mater. 2005, 17, 91-96.

[4] Mikulec, F. V.; Kuno, M.; Bennati, M.; Hall, D. A.; Griffin, R. G.; Bawendi, M. G. Organometallic synthesis and spectroscopic characterization of manganese-doped CdSe nanocrystals. J. Am. Chem. Soc. 2000, 122, 25322540.

[5] Hanif, K. M.; Meulenberg, R. W.; Strouse, G. F. Magnetic ordering in doped $\mathrm{Cd}_{1-x} \mathrm{CO}_{x} \mathrm{Se}$ diluted magnetic quantum dots. J. Am. Chem. Soc. 2002, 124, 11495-11502.

[6] Radovanovic, P. V.; Gamelin, D. R. Electronic absorption spectroscopy of cobalt lons in diluted magnetic semiconductor quantum dots: Demonstration of an isocrystalline core/shell synthetic method. J. Am. Chem. Soc. 2001, 123, 12207-12214.

[7] Sapra, S.; Sarma, D. D.; Sanvito, S.; Hill, N. A. Influence of quantum confinement on the electronic and magnetic properties of ( $\mathrm{Ga}, \mathrm{Mn})$ as diluted magnetic semiconductor. Nano Lett. 2002, 2, 605-608.

[8] Yuhas, B. D.; Zitoun, D. O.; Pauzauskie, P. J.; He, R.; Yang, P. Transition-metal doped zinc oxide nanowires. Angew. Chem. Int. Ed. 2006, 45, 420-423.

[9] Yang, Y.; Chen, O.; Angerhofer, A.; Cao, Y. C. Radialposition-controlled doping in $\mathrm{CdS} / \mathrm{ZnS}$ core/shell nanocrystals. J. Am. Chem. Soc. 2006, 128, 1242812429.

[10] Pradhan, N.; Peng, X. Efficient and color-tunable Mndoped ZnSe nanocrystal emitters: Control of optical performance via greener synthetic chemistry. J. Am. Chem. Soc. 2007, 129, 3339-3347.
[11] Viswanatha, R.; Chakraborty, S.; Basu, S.; Sarma, D. D. Blue-emitting copper-doped zinc oxide nanocrystals. J. Phys. Chem. B 2006, 110, 22310-22312.

[12] Nag, A.; Sapra, S.; Nagamani, C.; Sharma, A.; Pradhan, N.; Bhat, S. V.; Sarma, D. D. A study of $\mathrm{Mn}^{2+}$ doping in CdS nanocrystals. Chem. Mater. 2007, 19, 3252-3259.

[13] Peng, X. Mechanisms for the shape-control and shapeevolution of colloidal semiconductor nanocrystals. Adv. Mater. 2003, 15, 459-463.

[14] Peng, X.; Manna, U.; Yang, W.; Wickham, J.; Scher, E.; Kadavanich, A.; Allvisatos, A. P. Shape control of CdSe nanocrystals. Nature 2000, 404, 59-61.

[15] Manna, L.; Milliron, D. J.; Meisel, A.; Scher, E. C.; Alivisatos, A. P. Controlled growth of tetrapod-branched inorganic nanocrystals. Nat. Mater. 2003, 2, 382-385.

[16] Li, L. S.; Pradhan, N.; Wang, Y.; Peng, X. High quality ZnSe and ZnS nanocrystals formed by activating zinc carboxylate precursors. Nano Lett. 2004, 4, 2261-2264.

[17] Yu, W. W.; Peng, X. Formation of high-quality CdS and other II-VI semiconductor nanocrystals in noncoordinating solvents: Tunable reactivity of monomers. Angew. Chem. Int. Ed. 2002, 41, 2368-2371.

[18] Chen, Y.; Kim, M.; Lian, G.; Johnson, M. B.; Peng, X. Side reactions in controlling the quality, yield, and stability of high quality colloidal nanocrystals. J. Am. Chem. Soc. 2005, 127, 13331-13337.

[19] Pradhan, N.; Reifsnyder, D.; Xie, R.; Aldana, J.; Peng, X. Surface ligand dynamics in growth of nanocrystals. $J$. Am. Chem. Soc. 2007, 129, 9500-9509. 